\title{
Aproximación a la racionalidad de la experiencia amorosa. Validación y relevancia del Modelo de Amor Racional.
}

\author{
José Carlos Vázquez Parra \\ Doctor en Estudios Humanísticos \\ Tecnológico de Monterrey en Guadalajara \\ Departamento de Estudios Humanísticos \\ jcvazquezp@itesm.mx
}

Recibido: 23 de mayo de 2017

Aceptado: 20 de julio de 2017

\begin{abstract}
The present article seeks to validate the theoretical and empirical relevance of the Rational Love Model proposed in the article The Rationality of Love. An analysis from the Elsterian Theory of the Rationality of Emotions, with the intention of demonstrating the suitability of the generation of rational love experiences. To achieve this, a mixed analysis of information has been made from a sample group of 72 couples, where the adequacy of their beliefs is contrasted with the reality and the influence of the ideals with their notion of couple. As a result, it has been possible to identify that there is a clear tendency towards the idealization of the subject and object, which triggers clear differences between the objectives posed by the couples.
\end{abstract}

Keywords: Jon Elster, Rationality, Emotions, Love, Cognitive ideals.

\section{Resumen}

El presente artículo busca hacer una validación de la relevancia teórica y empírica del Modelo de Amor Racional propuesto en el artículo La Racionalidad del Amor. Un análisis desde la Teoría Elsteriana de la Racionalidad de las Emociones, con la intensión de demostrar la conveniencia de la generación de experiencias amorosas racionales. Para conseguirlo, se ha hecho un análisis mixto de información proveniente un grupo muestra de 72 parejas, en donde se contrasta la adecuación de sus creencias con la realidad y la influencia de los ideales con su noción de pareja. Como resultado, se ha conseguido identificar que existe una clara tendencia a la idealización del sujeto y objeto, lo que desencadena claras diferencias entre los objetivos planteados por las parejas.

Palabras Clave: Jon Elster, Racionalidad, Emociones, Amor, Ideales Cognitivos. 
Invest. pens. crit. (ISSN 1812-3864)

Vol. 5, No. 2, mayo-agosto 2017

pp. 37-52

\section{Introducción}

Estando en un mundo con casi 7 billones de habitantes, es sorprendente que uno de los problemas contemporáneos que más aqueja a millones sea la búsqueda de una pareja. Basta con hacer un análisis de medios de comunicación para reconocer la enorme industria que simboliza la búsqueda o el fracaso de la experiencia amorosa, lo que genera un cúmulo exagerado de ideales que nublan la objetividad de lo que comprendemos hoy en día por amor.

Según Galimberti (2002), el amor es una relación dual que se basa en un intercambio emotivo de diversa intensidad y duración, originado por dos necesidades básicas: La satisfacción sexual y el intercambio afectivo. Sin embargo, aunque Galimberti y otros autores que vienen desde Descartes (Kambouchner, 2007), hasta contemporáneos como Singer (1999), señalen esta dualidad de la experiencia amorosa, es importante que consideremos que en el momento en que un individuo se enamora este desdoblamiento se funde en uno, dando pauta a una sola emoción que conocemos como enamoramiento o amor romántico.

Aunque se han dedicado millones de palabras y miles de textos para hablar de este tema, se considera que son los menos los que se enfocan en plantear una explicación objetiva de este proceso emotivo, ya que en su mayoría lo que se escribe del amor es en torno al ideal de la experiencia, es decir, lo que se esperaría de un "buen" amor, y no así del proceso racional que se lleva a cabo por el agente que se enamora. Por ello es que se justifica la conveniencia de seguir reflexionando sobre este tema, ya que mientras el ser humano tenga la necesidad de amar, siempre se podrá escribir o señalar algo al respecto.

Con base en lo anterior, y tomando como referencia el artículo La Racionalidad del Amor. Un análisis desde la Teoría Elsteriana de la Racionalidad de las Emociones (Vázquez, 2016) el presente texto busca hacer una validación de la relevancia teórica y empírica del Modelo de Amor Racional (MAR) propuesto, con la intensión de demostrar la conveniencia de la generación de experiencias amorosas racionales. Para conseguirlo, se ha hecho un análisis mixto de información proveniente un grupo muestra de 72 parejas, en donde se contrasta la adecuación de sus creencias con la realidad y la influencia de los ideales con su noción de pareja.

\section{Marco Teórico}

\subsection{El amor romántico}

El amor, más que ser comprendido como una emoción aislada, debe ser visto como un conjunto de sentimientos interrelacionados que surgen entre dos agentes individuales que de manera libre e intencional buscan compartir sus objetivos de vida. Por ello, es que existen múltiples precisiones sobre el tipo de amor que corresponde a cada tipo de agente, así como a la relación que nace entre ellos.

Lewis (2005) considera que la reciprocidad esperada entre los individuos es una característica que diferencia los tipos de amor, y por ello plantea que no es lo mismo un amor filiar (philia), que un amor erótico (eros), o que incluso, que una expresión de ágape. Para Lewis, la philia exige que los agentes tengan una reciprocidad entre lo que aportan a la relación, requiriendo un equilibrio entre lo que uno espera del otro. Esto no se presenta en el eros, el cual, motivado 
mayormente por el deseo, no teme romper con la moderación y la armonía con tal de cubrir sus necesidades de satisfacción sexual. Un caso completamente distinto se da con el ágape, el cual tampoco tiende a una reciprocidad entre los enamorados, sino más bien, a una entrega total con la esperanza de que el otro responda de la misma forma y así alcanzar la plenitud (Canto-Sperber, 2001). Aunque pareciera que el ágape debiese constituirse como la base del amor ideal, ni éste, ni el eros pueden tomarse como los más óptimos desde un enfoque de racionalidad, ya que el hecho de no estar constituidos a partir de una relación de igualdad y equilibrio, hace fácilmente cuestionable el que los agentes realmente puedan satisfacer objetivamente sus expectativas. No se duda que el ágape pueda llegar a ser el objetivo último de toda experiencia amorosa, en donde ambos individuos se entreguen en totalidad al otro, sin embargo, esto no es adecuado, si primero no nace de una relación armoniosa y satisfactoria para ambos agentes.

Sin embargo, es inevitable que en algunos de los casos la experiencia amorosa pueda corromperse en el proceso de constitución, al momento en que alguno de los individuos genera creencias sesgadas o erróneas de la pareja a causa de un sentido de urgencia o emotividad desbordada. Esta situación distorsiona severamente la experiencia del enamoramiento, confundiendo lo ideal con lo real, ya sea en el individuo sujeto del amor, o incluso, de la noción o concepto concreto de lo que la experiencia amorosa significa.

Una relación que nace de un error cognitivo tiene un futuro bastante incierto, ya que basta con que el agente haga consciente la distorsión para que éste reconozca que lo que siente no es real y que la decisión de unirse al otro tal vez fue demasiado anticipada. Elster (2001) plantea que una persona con una racionalidad distorsionada puede llegar a casarse por un sentido irreal de urgencia, el cual no le permite descubrir las verdaderas cualidades de la pareja, sean estas adecuadas para motivar el matrimonio o contrarias a lo que se esperaría de una pareja para toda la vida.

De esta forma, es que es posible apreciar como el amor y la razón muchas ocasiones no resultan trabajar de la misma manera, pero que si lo hicieran, serían una excelente pareja. Desde este punto es donde se surge la necesidad de un amor racional, mismo que al constituirse entre dos agentes libres y racionales, esperaría como resultado la generación de un comportamiento con las mismas características.

\section{$2.2 \mathrm{El}$ amor racional}

Jon Elster, sociólogo noruego especialista en el análisis de la racionalidad humana, ha dedicado gran parte de sus escritos al estudio de las emociones y como éstas juegan un papel determinante en el proceso de racionalidad de los agentes. Así, plantea que no debemos confundir las emociones con los sentimientos, ya que las emociones tienen claros antecedentes cognitivos que las configuran objetos intencionales, y por ende, la racionalidad forma parte de sus procesos de estructuración y expresión (Elster, 2002).

La noción de la racionalidad de las emociones, puede resultar confrontante ya que usualmente éstas se relacionan con conductas meramente irracionales, sin embargo, desde el momento en que la intencionalidad forma parte las mismas, las emociones gozan de un vínculo con el comportamiento lo que justifica la posibilidad de analizarse desde una teoría de la acción (Elster, 2007).

Las emociones, al igual que los actos, tienen una base racional, sin embargo, son los factores externos de los procesos individuales de generación de creencias, los que las llevan a ver afectada su objetividad. Hablar de una emoción racional, es concebir una emotividad que se respalde por información y evidencia óptima, es decir, que no sea motivada por factores subjetivos 
Invest. pens. crit. (ISSN 1812-3864)

Vol. 5, No. 2, mayo-agosto 2017

pp. 37-52

que afectan la objetividad misma de lo que se siente. La racionalidad de la emoción no radica en la emoción en sí misma, sino en los procesos causales que llevan al individuo a experimentar ese conjunto de sentimientos de la forma en que los percibe.

De tal manera, es que el amor no se apega únicamente a aquello que objetivamente comprendemos por enamoramiento, sino que también se ve influido por factores como las creencias del agente, su entorno social, o incluso, los valores personales y culturales con los que ha sido formado. Por ello es que al momento de hablar de amor racional es menester que se profundice en las líneas causales de la experiencia amorosa, ya que solo así se puede concluir que la emoción es objetivamente confiable.

Elster (2002) contempla que para poder llevar a cabo un análisis de la racionalidad de las emociones, en este caso del amor, es necesario que se consideren de manera especial las creencias sobre las que el agente se sustenta para desarrollar ese comportamiento, ya que las emociones racionales tienen una clara relación con la generación de creencias óptimas, por lo que el estudio de las líneas causales de éstas, amerita inevitablemente el escrutinio del proceso constitutivo de las otras.

El agente que es motivado por cierto deseo que tiene por objetivo el desarrollar la emoción del amor hacia un sujeto, debe prever que las creencias que respalden dicho comportamiento sean lo más racionalmente posibles, pues solo de esta forma puede generarse una experiencia amorosa satisfactoria (Vázquez, 2016). Por creencias racionales se entiende a aquellas creencias que, basadas en evidencia óptima, se tienen acerca del objeto (la concepción del amor) y el sujeto que despierta la emoción, mismas que resultan fundamentales al momento de realizar cualquier comportamiento que contribuya o atente con la experiencia amorosa.

De esta manera, es que se puede comprender el por qué analizar la racionalidad de las emociones a partir de sus elementos constitutivos puede permitir hacer una aproximación a una concepción mucho más racional y menos incierta del fenómeno amoroso; lo cual, visto desde una perspectiva estructural, no es más que otro proceso comportamental, como lo sería el buscar trabajo o elegir un lugar para comer, aunque a diferencia de estos, se acompaña de una pulsión emocional que si no es controlada por la razón, puede cegar plenamente la objetividad. Bajo este enfoque, se ve al amor no simplemente como un sentimiento motivador de una experiencia amorosa, sino más bien, como todo un proceso comportamental, que vale la pena analizar de forma independiente y con una herramienta propia.

\subsection{El Modelo de Amor Racional (MAR)}

Amar racionalmente, comprende la necesidad de que el agente ponga especial atención a las líneas causales de la emoción, evaluando las creencias que respaldan su concepción del amor y considerando que la información que se ha recabado del objeto de deseo sea una evidencia realmente confiable (Vázquez, 2016). Por ello es que el presente artículo busca validar la relevancia que puede llegar a tener el Modelo de Amor Racional (Figura 1) que se ha propuesto en un anterior texto del autor, por considerar que es una excelente herramienta de responder a los cuestionamientos que cognitivamente se suelen hacer acerca de la emoción del amor, por considerarla una emotividad sesgada, ciega, desbordada y peligrosamente irracional. 
Figura 1: Modelo de Amor Racional (MAR)

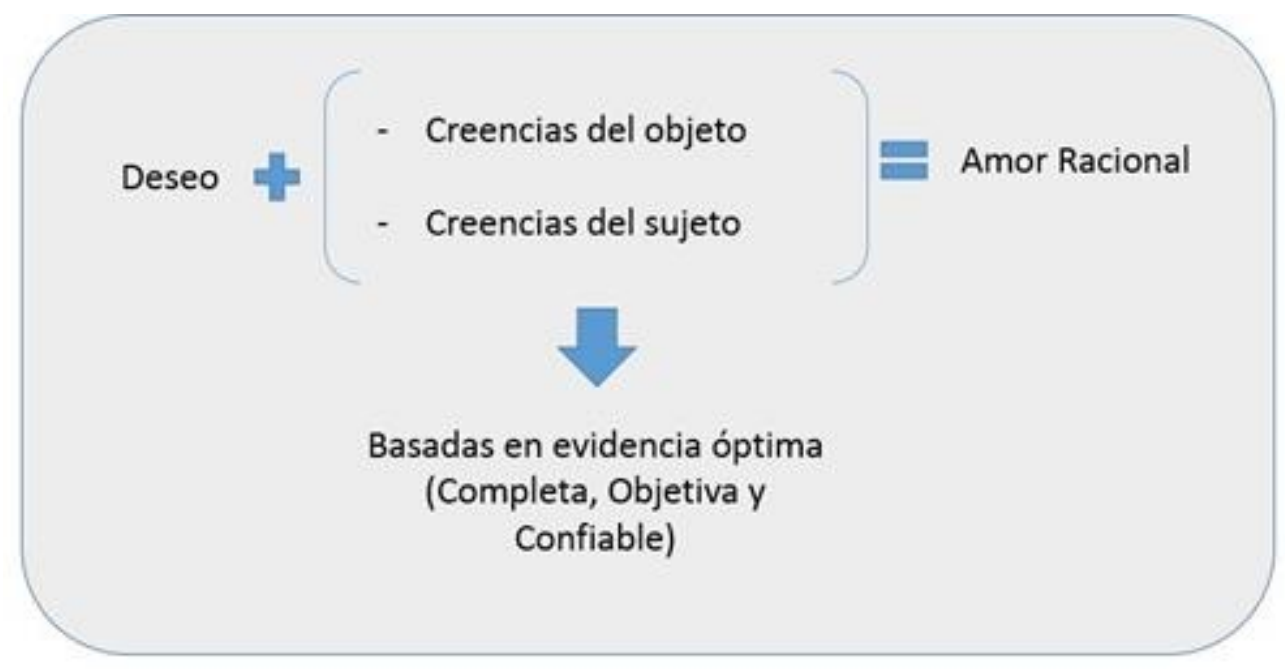

Fuente: (Vázquez, 2016)

El MAR se configura como una herramienta que permite exponer de una manera mucho más certera y precisa, lo que idealmente debería entenderse como proceso de enamoramiento, es decir, el lapso de tiempo en el que los individuos se conocen, familiarizan uno con el otro y deciden racionalmente formar parte de una experiencia amorosa. De manera adicional, este modelo permite, no únicamente configurar una emoción mucho más óptima y objetiva, sino que también evita en gran medida, el claro poder distorsionador que como puede llegar a tener el enamoramiento, el deseo posesivo y el amor ciego.

La posibilidad de plantear una herramienta que marque el camino más idóneo para la experiencia amorosa permite que los individuos puedan enfocarse realmente en el cumplimiento de los objetivos comunes que tiene la pareja, evitando perder la perspectiva y el rumbo inicial que los llevó a estar juntos.

Como se ha señalado con anterioridad, el presente artículo tiene el objetivo de validar el Modelo de Amor Racional (MAR) con la intensión de demostrar la conveniencia de generar experiencias amorosas racionales. Para conseguirlo se ha hecho un estudio empírico, mismo que se presenta a continuación.

\section{Estudio empírico.}

Con base en el breve marco teórico anterior, es posible justificar la relevancia de validar empíricamente el MAR, ya que no basta con la propuesta de un modelo si no se hace un análisis de la aplicabilidad y beneficios del mismo. Aunque Elster (2007), considera que la racionalización plena de las emociones no es un proceso sencillo de realizar, no es posible que se abandone la posibilidad de alcanzar una mayor objetividad de la emoción misma, por medio de una estructura cognitiva que permita tener una mayor certidumbre de la experiencia de enamoramiento. Así, el estudio empírico se ha guiado a partir de los siguientes cuestionamientos, ¿Qué relación existe entre los parámetros propuestos por el MAR y la experiencia amorosa vivida por un grupo de parejas?, ¿Habrá diferencias significativas entre aquellas parejas que cuentan con creencias objetivas sobre el sujeto y objeto amoroso, y aquellas que sustentan sus creencias en ideales subjetivos?, ¿Cuáles son las consecuencias que puede traer consigo una falta de racionalidad en el proceso de enamoramiento? 
Invest. pens. crit. (ISSN 1812-3864)

Vol. 5, No. 2, mayo-agosto 2017

pp. 37-52

\subsection{Hipótesis}

Tomando en cuenta que el estudio se ha realizado con base en una metodología cuantitativa, se generaron una serie de hipótesis relacionando algunos señalamientos previos de la teoría Elsteriana y el MAR. De esta forma, las hipótesis de la investigación se plantearon de la siguiente manera:

\section{Hipótesis 1.}

Elster (2002) considera que el mayor problema de las emociones irracionales es que estas pueden afectar rotundamente la percepción que tenemos de la realidad generando comportamientos o pensamientos fantasiosos o irracionales. Esta situación ha permitido que Elster señale que la irracionalidad puede dar pauta a comportamientos igualmente limitados de razón entre los que usualmente se encuentran las reacciones ilegítimas (reacciones emocionales sin una justificación real), las divergencias entre objeto y causa (emociones injustificadas basadas en causas incontrolables por el agente), divergencias emocionales entre grado y fuerza (reacciones con una intensidad que no corresponde al estímulo real de la emoción) y la afectación por otras emociones, deseos o creencias irracionales. Por ende:

H1. La presencia de comportamientos o pensamientos fantasiosos o irracionales sobre el ser y los objetos de la relación puede generar factores que detonen diferencias o problemas en el desarrollo de la pareja.

\section{Hipótesis 2 y 3}

Con base en la propuesta Elsteriana, se entiende como creencia racional aquella creencia que, basada en evidencia óptima, se tiene acerca del objeto (la concepción del amor) y del sujeto que despierta la emoción (Elster, 2001). Esto lleva a pensar que una creencia errónea, perversa o sesgada de la concepción que se tiene sobre el amor puede llevar a la generación de comportamientos errados o arbitrarios, que pudieran ser contradictorios a las metas de la pareja, ya que se sustentan en un ideal que no es objetivamente realista con la relación que se vive. De igual manera puede suceder cuando se tienen creencias equivocadas o confusas de la persona, lo que puede dar pauta a procesos de idealización del individuo que resulten problemáticos cuando el comportamiento de la pareja no se apegue a lo que se espera idealmente de ella (Vázquez, 2016). Por ende:

H2. Las parejas con creencias irracionales sobre el objeto (concepción del amor) son más propensas a generar comportamientos contradictorios y con ello diferencias sobre los objetivos fijados por la pareja.

H3. Las parejas con creencias irracionales sobre el sujeto son más propensas a generar una imagen idealizada y con ello una disonancia cognitiva sobre lo que se espera de la pareja.

\subsection{Metodología}

El estudio empírico realizado se implementó a partir de un análisis con dos perspectivas; un apartado cuantitativo que se enfoca exclusivamente en la contabilización de los datos recabados, y un apartado cualitativo, que permitió hacer una reflexión más profunda sobre las respuestas dadas por la población muestra. El estudio abundó en la medición del grado de relación existente entre 
la calidad racional de las creencias que tienen las parejas seleccionadas y posibles comportamientos o reacciones irracionales que han presentado durante su experiencia de enamoramiento.

Considerando que el estudio busca señalar una relación entre variables, éste ha medido primeramente dos variables primarias; creencias racionales y comportamientos o reacciones irracionales, así como posteriormente 7 variables secundarias; reacciones ilegitimas, divergencias entre objeto y causa, divergencias emocionales entre grado y fuerza, afectación por deseos, creencias o emociones irracionales, comportamientos contradictorios con los objetivos fijados por la pareja, idealización de la relación e idealización del sujeto amado.

El hecho de haber elegido una metodología mixta permite no únicamente tener una aproximación observable a la medida en que las variables se encuentran relacionadas, sino que también brinda la oportunidad de considerar cualquier otra información que pudiera surgir durante la interacción con la población muestra.

\subsection{Población}

El estudio fue realizado en una población muestra de 72 parejas, que incluyen 65 heterosexuales y 7 homosexuales ( 5 varón-varón y 3 mujer-mujer), considerando como criterios de inclusión el que estuvieran en un rango de edad entre los 20 y 25 años, que tuvieran menos más de 3 meses y menos de 1 año de estar juntos, que no vivieran juntos, que no estuvieran casados, que no tuvieran hijos, que fueran de nacionalidad mexicana y que después del conteo final hubiera un equilibrio de género (71 mujeres y 73 hombres). Es importante mencionar que 2 parejas fueron excluidas de la población original porque uno de los miembros tenía una edad superior a la del criterio de inclusión, así como 1 pareja más que tuvo que excluirse por sobrepasar el tiempo de relación.

El estudio se realizó en dos universidades privadas del municipio de Zapopan en el Estado de Jalisco, México, considerando que las características socioeconómicas y de formación de ambos centros de estudio contaban con parámetros similares y que no representarían diferencias significativas entre la población muestra.

\subsection{Instrumento de medición}

Para los fines de la presente investigación se utilizaron dos instrumentos de medición: una encuesta individual con parámetros de medición con escala tipo Likert y un conjunto de 5 dilemas complementarios para la resolución en pareja. Mientras el cuestionario será analizado a partir de los resultados arrojados, la reflexión de los dilemas se hará a partir de un proceso de observación y reflexión de cada una de las parejas concretas con base en sus resultados individuales.

La encuesta individual se ha estructurado con base en los 7 variables objetos de la medición, considerando 10 preguntas que permitan plantear un horizonte sobre la presencia de creencias irracionales en cada individuo (Tabla 1): 
Invest. pens. crit. (ISSN 1812-3864)

Vol. 5, No. 2, mayo-agosto 2017

pp. 37-52

Tabla 1: Instrumento de medición de variables

\begin{tabular}{|c|c|c|c|c|c|}
\hline \multirow[b]{2}{*}{$\begin{array}{l}\text { Pregunta } \\
\text { 1. ¿Qué tan seguido tú o tu pareja } \\
\text { tienen diferencias a causa de } \\
\text { reacciones emocionales (enojo, } \\
\text { llanto, nervios, celos, etc.) sin } \\
\text { razón aparente o sin una } \\
\text { justificación? }\end{array}$} & Siempre & Usualmente & A veces & Casi nunca & Nunca \\
\hline & & & & & \\
\hline $\begin{array}{l}\text { 2. ¿Qué tan seguido tú o tu pareja } \\
\text { tienen diferencias a causa de } \\
\text { reacciones emocionales (enojo, } \\
\text { llanto, nervios, celos, etc.) a causa } \\
\text { de algo que es incontrolable, ajeno } \\
\text { o que no depende de ustedes? }\end{array}$ & & & & & \\
\hline $\begin{array}{l}\text { 3. ¿Qué tan seguido tú o tu pareja } \\
\text { tienen diferencias a causa de } \\
\text { reacciones emocionales (enojo, } \\
\text { llanto, nervios, celos, etc.) } \\
\text { desproporcionadas en relación } \\
\text { con lo que las ha provocado? }\end{array}$ & & & & & \\
\hline $\begin{array}{l}\text { 4. ¿Qué tan seguido tú o tu pareja } \\
\text { tienen diferencias por creencias o } \\
\text { reacciones emocionales sin un } \\
\text { verdadero fundamento, causa o } \\
\text { motivo real, cierto o justificado? }\end{array}$ & & & & & \\
\hline $\begin{array}{l}\text { 5. ¿Qué tan seguido tú o tu pareja } \\
\text { tienen diferencias a causa de } \\
\text { comportamientos contradictorios } \\
\text { a las decisiones u objetivos } \\
\text { planteados por la pareja en } \\
\text { conjunto? }\end{array}$ & & & & & \\
\hline $\begin{array}{l}\text { 6. ¿Qué tan seguido tú o tu pareja } \\
\text { tienen diferencias a causa de } \\
\text { nociones desiguales sobre lo que } \\
\text { es o no es una relación de pareja? }\end{array}$ & & & & & \\
\hline $\begin{array}{l}\text { 7. ¿Qué tan seguido tú o tu pareja } \\
\text { tienen diferencias a causa de } \\
\text { incongruencias entre lo que hace o } \\
\text { se espera del otro? }\end{array}$ & & & & & \\
\hline $\begin{array}{l}\text { 8. ¿Qué tan seguido tú o tu pareja } \\
\text { tienen diferencias a causa de un } \\
\text { desconocimiento sobre lo que el } \\
\text { otro espera o valora? }\end{array}$ & & & & & \\
\hline $\begin{array}{l}\text { 9. ¿Qué tan seguido tú o tu pareja } \\
\text { tienen diferencias a causa de una } \\
\text { discordancia entre los objetivos } \\
\text { individuales y los de la pareja? }\end{array}$ & & & & & \\
\hline
\end{tabular}


10. ¿Qué tan seguido tú o tu pareja tienen diferencias a causa de una divergencia entre los planes a futuro?

Fuente: Creación propia

El hecho de que las preguntas hagan referencia a diferencias entre el sujeto encuestado y su pareja se da con la intensión de identificar situaciones que pudieran resultar conflictivas por no estar apegadas a la realidad o a información objetiva. Cabe señalar que la presencia de creencias irracionales en alguno de los miembros de la relación es un elemento que usualmente puede generar un factor de diferencias en la pareja, ya que no puede haber congruencia y equilibrio cuando uno de los individuos tiene una percepción de la realidad o del sujeto distorsionada a lo que objetivamente es.

De manera adicional a los resultados individuales, se plantea la reflexión de una serie de dilema que busquen confrontar la percepción de la pareja con la intensión de identificar aquellos factores que resultan más controversiales. Para su medición se ha generado un instrumento con aquellos casos que ameriten el que la pareja deba tomar una decisión en conjunto, en torno a problemas o situaciones hipotéticas que usualmente se podrían presentar en una relación dentro de los primeros meses (Tabla 2). Los 5 casos seleccionados para el análisis, discusión y resolución por las parejas de la población muestra se han tomado considerando los resultados de la encuesta individual, así como el ciclo de las relaciones propuesto por Vázquez (2012) en su libro Créelo, tú puedes tener pareja y encontrar el amor.

Tabla 2: Dilemas para la medición de variables

\begin{tabular}{|l|l|}
\hline $\begin{array}{l}\text { 1. ¿Cuál es el ciclo "natural” de una relación } \\
\text { (incluye tiempos probables)? }\end{array}$ & $\begin{array}{l}\text { Comportamientos contradictorios con los } \\
\text { objetivos fijados por la pareja }\end{array}$ \\
\hline $\begin{array}{l}\text { 2. ¿Qué tan importante es la convivencia con el } \\
\text { grupo social o familiar en la relación? }\end{array}$ & Idealización de la relación o la pareja \\
\hline $\begin{array}{l}\text { 3. ¿Qué harían en caso de una mentira o } \\
\text { infidelidad dentro de la relación? }\end{array}$ & Idealización de la pareja \\
\hline $\begin{array}{l}\text { 4. ¿Qué tanto tiempo requiere una pareja para } \\
\text { poder formalizar la relación (vivir juntos, } \\
\text { casarse, etc.)? }\end{array}$ & Idealización de la relación \\
\hline $\begin{array}{l}\text { 5. ¿Qué harían como pareja si a mediano o largo } \\
\text { plazo alguno tuviera que mudarse a otra ciudad } \\
\text { o país inevitablemente? }\end{array}$ & Divergencia entre objeto y causa \\
\hline
\end{tabular}

Fuente: Creación propia (Vázquez, 2012)

\section{Resultados del estudio}

Los primeros resultados que se tuvieron de la población muestra fueron los correspondientes al instrumento para la medición de las variables con impacto individual, mismo que se aplicó durante los meses de agosto a octubre del 2016. Para tener una mayor claridad sobre los datos se presenta la siguiente tabla (Tabla 3), en donde se han colocado las variables junto con la o las preguntas que se relacionan con las mismas y los resultados arrojados por la población muestra, en cantidad y su porcentaje. 
Invest. pens. crit. (ISSN 1812-3864)

Vol. 5, No. 2, mayo-agosto 2017

pp. 37-52

Tabla 3: Resultados del instrumento según variable (cantidad y porcentaje)

\begin{tabular}{|c|c|c|c|c|c|c|}
\hline Variable & Pregunta & Siempre & Usualmente & $\begin{array}{l}\text { A } \\
\text { veces }\end{array}$ & $\begin{array}{l}\text { Casi } \\
\text { nunca }\end{array}$ & Nunca \\
\hline $\begin{array}{l}\text { Reacciones } \\
\text { ilegítimas }\end{array}$ & $\begin{array}{l}\text { 1. ¿Qué tan seguido tú } \\
\text { o tu pareja tienen } \\
\text { diferencias a causa de } \\
\text { reacciones } \\
\text { emocionales (enojo, } \\
\text { llanto, nervios, celos, } \\
\text { etc.) sin razón } \\
\text { aparente o sin una } \\
\text { justificación? }\end{array}$ & $5(4)$ & $21(15)$ & $\begin{array}{l}46 \\
(32)\end{array}$ & $63(44)$ & $9(6)$ \\
\hline $\begin{array}{l}\text { Divergencias } \\
\text { entre objeto y } \\
\text { causa }\end{array}$ & $\begin{array}{l}\text { 2. ¿Qué tan seguido tú } \\
\text { o tu pareja tienen } \\
\text { diferencias a causa de } \\
\text { reacciones } \\
\text { emocionales (enojo, } \\
\text { llanto, nervios, celos, } \\
\text { etc.) a causa de algo } \\
\text { que es incontrolable, } \\
\text { ajeno o que no } \\
\text { depende de ustedes? }\end{array}$ & 0 & $2(1)$ & $\begin{array}{l}38 \\
(26)\end{array}$ & $22(15)$ & $\begin{array}{l}82 \\
(57)\end{array}$ \\
\hline $\begin{array}{l}\text { Divergencias } \\
\text { emocionales entre } \\
\text { grado y fuerza }\end{array}$ & $\begin{array}{l}\text { 3. ¿Qué tan seguido tú } \\
\text { o tu pareja tienen } \\
\text { diferencias a causa de } \\
\text { reacciones } \\
\text { emocionales (enojo, } \\
\text { llanto, nervios, celos, } \\
\text { etc.) } \\
\text { desproporcionadas en } \\
\text { relación con lo que las } \\
\text { ha provocado? }\end{array}$ & $9(6)$ & $36(25)$ & $\begin{array}{l}53 \\
(37)\end{array}$ & $38(26)$ & $8(6)$ \\
\hline $\begin{array}{l}\text { Afectación por } \\
\text { deseos, creencias } \\
\text { o emociones } \\
\text { irracionales }\end{array}$ & $\begin{array}{l}\text { 4. ¿Qué tan seguido tú } \\
\text { o tu pareja tienen } \\
\text { diferencias por } \\
\text { creencias o reacciones } \\
\text { emocionales sin un } \\
\text { verdadero } \\
\text { fundamento, causa o } \\
\text { motivo real, cierto o } \\
\text { justificado? }\end{array}$ & 0 & $1(1)$ & $6(4)$ & $36(25)$ & $\begin{array}{l}101 \\
(70)\end{array}$ \\
\hline $\begin{array}{l}\text { Comportamientos } \\
\text { contradictorios } \\
\text { con los objetivos } \\
\text { fijados por la } \\
\text { pareja }\end{array}$ & $\begin{array}{l}\text { 5. ¿Qué tan seguido tú } \\
\text { o tu pareja tienen } \\
\text { diferencias a causa de } \\
\text { comportamientos } \\
\text { contradictorios a las } \\
\text { decisiones u objetivos }\end{array}$ & $1(1)$ & $1(1)$ & $4(3)$ & $41(28)$ & $\begin{array}{l}97 \\
(67)\end{array}$ \\
\hline
\end{tabular}




\begin{tabular}{|c|c|c|c|c|c|c|}
\hline & $\begin{array}{l}\text { planteados por la } \\
\text { pareja en conjunto? }\end{array}$ & & & & & \\
\hline $\begin{array}{l}\text { Idealización de la } \\
\text { relación }\end{array}$ & $\begin{array}{l}\text { 6. ¿Qué tan seguido tú } \\
\text { o tu pareja tienen } \\
\text { diferencias a causa de } \\
\text { nociones desiguales } \\
\text { sobre lo que se espera } \\
\text { de una relación de } \\
\text { pareja? }\end{array}$ & $8(6)$ & $32(22)$ & $\begin{array}{l}76 \\
(52)\end{array}$ & $28(20)$ & 0 \\
\hline $\begin{array}{l}\text { Idealización de la } \\
\text { relación }\end{array}$ & $\begin{array}{l}\text { 7. ¿Qué tan seguido tú } \\
\text { o tu pareja tienen } \\
\text { diferencias a causa de } \\
\text { incongruencias entre } \\
\text { lo que hace o se espera } \\
\text { de la relación a } \\
\text { mediano o largo } \\
\text { plazo? }\end{array}$ & 7 (6) & $41(28)$ & $\begin{array}{l}58 \\
(40)\end{array}$ & $28(19)$ & $10(7)$ \\
\hline $\begin{array}{l}\text { Idealización de la } \\
\text { pareja }\end{array}$ & $\begin{array}{l}\text { 8. ¿Qué tan seguido tú } \\
\text { o tu pareja tienen } \\
\text { diferencias a causa de } \\
\text { un desconocimiento } \\
\text { sobre lo que el otro } \\
\text { espera o valora? }\end{array}$ & $10(7)$ & $72(50)$ & $\begin{array}{l}46 \\
(32)\end{array}$ & $15(10)$ & $1(1)$ \\
\hline $\begin{array}{l}\text { Idealización de la } \\
\text { pareja }\end{array}$ & $\begin{array}{l}\text { 9. ¿Qué tan seguido tú } \\
\text { o tu pareja tienen } \\
\text { diferencias a causa de } \\
\text { una discordancia entre } \\
\text { los objetivos o deseos } \\
\text { individuales y los de la } \\
\text { pareja? }\end{array}$ & $12(8)$ & $47(33)$ & $\begin{array}{l}49 \\
(34)\end{array}$ & $25(17)$ & $11(8)$ \\
\hline $\begin{array}{l}\text { Comportamientos } \\
\text { contradictorios } \\
\text { con los objetivos } \\
\text { fijados por la } \\
\text { pareja }\end{array}$ & $\begin{array}{l}\text { 10. ¿Qué tan seguido } \\
\text { tú o tu pareja tienen } \\
\text { diferencias a causa de } \\
\text { una divergencia entre } \\
\text { los planes a futuro? }\end{array}$ & $6(4)$ & $22(15)$ & $\begin{array}{l}42 \\
(30)\end{array}$ & $48(33)$ & $\begin{array}{l}26 \\
(18)\end{array}$ \\
\hline
\end{tabular}

Fuente: Creación propia

Como se puede apreciar en los resultados de la tabla (Tabla 3), la presencia de creencias irracionales individuales tiene una tendencia positiva únicamente en tres de las siete variables; idealización de la pareja, idealización de la relación y divergencias emocionales (aunque en esta última en menor escala), mientras que en el resto de los resultados la tendencia se mantiene entre la presencia muy esporádica o nula. Las variables con los resultados más bajos fueron las que tienen relación con la afectación por deseos, creencias o emociones irracionales, así como la de comportamientos contradictorios con los objetivos fijados por la pareja.

Aunque el resto de las variables no presentan una tendencia tan marcada como las anteriormente mencionadas, los resultados siguen siendo bastante significativos, ya que aunque sea en menor o mayor medida, es relevante notar que las actitudes, reacciones o creencias irracionales se encuentran presentes en la mayoría de los encuestados. Se puede observar que la idealización es 
Invest. pens. crit. (ISSN 1812-3864)

Vol. 5, No. 2, mayo-agosto 2017

pp. 37-52

el mayor problema que se presenta como factor generador de diferencias entre las parejas, ya sea en cualquiera de sus dos vertientes, relación o pareja, lo cual es congruente con el MAR al señalar que la distorsión en el objeto o sujeto suelen ser creencias que no permiten una experiencia amorosa objetiva. Esta situación marcó el camino que seguiría la aplicación del segundo instrumento, buscando precisar de una manera más certera la presencia de estos factores de idealización y las posibles consecuencias de los mismos en la relación. Toda esta información se profundizará en el apartado de análisis y discusión de los resultados.

En cuanto al segundo instrumento de recolección de información, éste ha permitido que se recaben muy pocos datos de carácter cuantitativo, pues el proceso de decisión de cada una de las parejas presentó rasgos tan propios que se ha preferido manejar una metodología cualitativa para analizar esta información. Es importante considerar que el objetivo de este instrumento es la contratación de las creencias de los miembros de la relación, así que independientemente de la decisión tomada, lo relevante era el poder identificar cuando los individuos confrontaban la realidad con lo que idealmente creían que la pareja o la relación era o debía ser, buscando ser un complemente de los datos recabados en la encuesta. Aun así, se presenta la siguiente tabla con algunos de los datos cuantificables recabados durante el mes de noviembre del 2016 a partir de hojas de observación que se recabaron durante la resolución de los dilemas en pareja (Tabla 4):

Tabla 4: Observaciones cuantificables de la resolución de dilemas en pareja.

\begin{tabular}{|c|c|c|c|c|c|}
\hline $\begin{array}{l}\text { Tiempo promedio en resolver los } 5 \text { dilemas } \\
\text { (Cantidad-Porcentaje) }\end{array}$ & $\begin{array}{l}\text { Menos } \\
\text { de } 5 \\
\min .(0)\end{array}$ & $\begin{array}{l}5-10 \\
\text { min. } \\
(41- \\
28)\end{array}$ & $\begin{array}{l}10-15 \\
\text { min. } \\
(69- \\
48)\end{array}$ & $\begin{array}{l}15-20 \\
\text { min. } \\
(32- \\
22) \\
\end{array}$ & $\begin{array}{l}\text { Más de } \\
20 \text { min. } \\
(2-2)\end{array}$ \\
\hline $\begin{array}{l}\text { Dilema en el que tardaron más tiempo en } \\
\text { promedio (Cantidad-Porcentaje) }\end{array}$ & $\begin{array}{l}\text { D.1 } \\
(52-36)\end{array}$ & $\begin{array}{l}\text { D.2 } \\
(24- \\
17) \\
\end{array}$ & $\begin{array}{l}\text { D.3 } \\
(4-3)\end{array}$ & $\begin{array}{l}\text { D.4 } \\
(46- \\
32) \\
\end{array}$ & $\begin{array}{l}\text { D.5 } \\
(18-13)\end{array}$ \\
\hline $\begin{array}{l}\text { Dilema en el que surgieron más diferencias } \\
\text { (Cantidad-Porcentaje) }\end{array}$ & $\begin{array}{l}\text { D.1 } \\
(68-47)\end{array}$ & $\begin{array}{l}\text { D.2 } \\
(15- \\
10)\end{array}$ & $\begin{array}{l}\text { D.3 } \\
(1-1)\end{array}$ & $\begin{array}{l}\text { D.4 } \\
(55- \\
38)\end{array}$ & $\begin{array}{l}\text { D.5 } \\
(5-4)\end{array}$ \\
\hline
\end{tabular}

Fuente: Creación propia

Con base en los resultados cuantificables que se pudieron recabar del segundo instrumento se puede apreciar que en promedio las parejas dedicaron un aproximado de entre 2 y 3 minutos para resolver cada uno de los dilemas, aunque es claro que hubo algunos de ellos que les resultaron especialmente complejos, como fue el caso de los dilemas 1 y 4 . De manera proporcional, estos también resultaron ser los dilemas en los que se dieron las mayores diferencias entre los miembros de la pareja, mientras que en el caso de los dilemas 3 y 5 , no se presentó una situación que significara una dificultad al momento de tomar una decisión en pareja. Al igual que los resultados arrojados por la tabla 3, parece ser que efectivamente la presencia de creencias irracionales individuales en cuanto al objeto y sujeto dentro de la relación, muestra una tendencia hacia la manifestación de diferencias a causa de factores de idealización, lo que parece constituirse como una de las variables de irracionalidad más usual en las parejas que conformaron la población muestra. 
Como se ha señalado con anterioridad la mayor aportación de este segundo instrumento es la información que se ha recabado cualitativamente de cada pareja, misma que se abordará en el apartado de discusión y análisis de resultados con la intensión de generar una reflexión que sirva como complemento de los datos cuantitativos recabados de manera individual.

\section{Análisis y discusión de los resultados}

Elster (2002) ha mencionado en múltiples ocasiones que las emociones no necesariamente deben relacionarse de manera exclusiva con la irracionalidad, ya que en algunos casos, son las emociones las mejores herramientas para solventar problemas racionales, como es el caso del hiperracionalismo (Vázquez, 2016b). Sin embargo, de manera complementaria plantea que sin un buen sistema de control, la emotividad puede desbordarse lo que puede no únicamente nublar la racionalidad de los agentes, sino también, afectar las decisiones que tomen durante en ese periodo de distorsión cognitiva.

Como se ha podido apreciar en la tabla 3, la tendencia generalizada de la población fue a manifestar, en una u otra de sus variables, comportamientos o reacciones característicos de la presencia de creencias irracionales sobre la experiencia amorosa que se encuentran viviendo. Esto no resulta ser una sorpresa para la investigación, ya que era de esperarse que la percepción de la relación variaría según el individuo, sus vivencias, edad, cultura, educación, familia, entre otros elementos. No se puede pretender que se alcance una objetividad plena sobre lo que es o se espera de la relación o el sujeto amado dentro del primer año de estar juntos (elemento solicitado como factor de inclusión), por lo que esto, aunque nos muestra el horizonte de la muestra, no es el verdadero descubrimiento.

Algo que si es importante señalar es lo que ha sucedido con las dos variables de idealización (objeto-sujeto), las cuales mostraron una tendencia significativamente alta. Aunque, como se mencionó anteriormente, es inevitable la presencia de creencias infundadas o sesgadas, no se esperaría que estos datos fueran tan marcados en una y mucho menos dos de las variables, ya que esto es un punto que puede llegar a cuestionar los procesos por los que las parejas actuales llegan a "conocerse". El hecho de que la mayoría de los resultados individuales mostraran la presencia de diferencias en la pareja a causa de situaciones de idealización, hace pensar que los miembros de relación deberían enfocarse en conocer realmente a la persona con la que pretenden compartir su vida, ya que estos datos reflejan que realmente la mayoría de la muestra se ha enamorado de un ideal y no del individuo real que tienen a un lado.

Buscando precisar de una manera más puntual los resultados individuales, los datos recabados se han cruzado transversalmente con las observaciones del segundo instrumento (tabla 2), tomando en cuenta las encuestas de cada miembro de la pareja y la resolución de los dilemas de forma conjunta. Como era de esperarse, aquellas parejas que presentaban resultados más altos en la encuesta individual terminaban siendo los que también tenían la mayor cantidad de diferencias al resolver los dilemas propuestos y requerían la mayor cantidad de tiempo (tabla 4), llegando incluso, en algunos casos, a no alcanzar un acuerdo en común. Esto se diferenciaba de aquellos que tenían resultados bajos, los cuales conseguían rápidamente responder a lo solicitado, mostrando una afinidad entre el sentir de ambas partes.

En cuanto a cada variable, aquellas parejas que de manera conjunta mostraban resultados de idealización en el objeto, es decir en la noción de la relación, tuvieron muchos problemas para definir el ciclo natural de la pareja y el momento para formalizar, pues tenían ideas diferentes sobre lo que una relación significaba en su vida. Entre las observaciones más relevantes se dieron casos en los que una de las partes no tenía intenciones de casarse o bien, consideraban que el vivir juntos de manera previa sería lo ideal para realmente conocerse. De manera lógica, la presencia de una 
Invest. pens. crit. (ISSN 1812-3864)

Vol. 5, No. 2, mayo-agosto 2017

pp. 37-52

idealización sobre la relación puede convertirse en un detonante de discrepancias y problemas al momento de tomar decisiones, ya que uno de los objetivos de la pareja es compartir objetivos comunes, los cuales al ser diferentes, muestran un futuro poco alentador si esto no es solventado.

De una manera semejante, las parejas que mostraron resultados elevados en la idealización del sujeto, tuvieron que confrontar durante la resolución de los dilemas, que la respuesta esperada por parte de la pareja, no necesariamente fue la contestación dada. Diferencias sobre el rol o participación de la familia en la relación, la importancia de las amistades o incluso los deseos de quedarse en casa o ser autónomos, fueron algunas de las posturas que parece ser que las parejas desconocían entre ellos, lo que dio pauta a muchas diferencias entre el ideal y lo real. Para Freud (2001), esto es algo usual en cualquier relación, ya que considera que la etapa del enamoramiento es muy poco objetiva sobre la concepción del individuo amado, caracterizandose por un estado de idealización originado en gran medida por las tendencias sexuales reprimidas del agente. De esta manera, parece ser que gran parte de los individuos de la muestra sobrestimaron al sujeto del deseo, lo que podría incluso cuestionar si lo que sienten es objetivo o si la emoción solo se ha dado en virtud de complacer su propio deseo ideal.

Por consiguiente, se puede señalar que los resultados arrojados por la aplicación individual, permiten dilucidar una relación con las observaciones recabadas mediante el segúndo instrumento, mostrando cierta concordancia con lo propuesto por el MAR. Así, la presencia de factores de idealización sobre el sujeto y el objeto por parte de los agentes de una relación realmente dan pauta a diferencias y conflictos en la experiencia amorosa, no permitiendo estructurar lo que en su momento se ha propuesto como amor racional.

\section{Validación de la hipotesis}

La hipótesis H1 no es corroborada, ya que aunque algunos sujetos de la muestra mostraron comportamientos o pensamientos fantasiosos o irracionales sobre el ser o los objetos de la relación, estos no consiguieron marcar una considerable tendencia sobre el resto de las variables, lo que deja a esta hipótesis sin datos suficientes para ser comprobada.

Las hipótesis $\mathrm{H} 2$ y H3 son corroboradas, pues efectivamente la idealización del objeto y el sujeto resultaron ser los factores que no únicamente mostraron una mayor tendencia individual en la población muestra, sino que también fueron los que notoriamente desencadenaron mayores diferencias al momento de ser contrastados en los dilemas de pareja.

\section{Conclusiones y limitantes}

Como se ha señalado en el inicio del artículo, existe una clara tendencia dentro de la academia a analizar a las emociones desde un punto de partida plenamente irracional, considerando que éstas pueden perder su esencia si se incluyen dentro de un proceso de racionalización. Sin embargo, como se ha podido apreciar durante toda la investigación, tanto en el presente como en el anterior artículo (Vázquez, 2016), existen factores que justifican la relevancia de llevar a cabo este cometido, ya que como sucede en el caso de la experiencia amorosa, el abandono de las emociones a merced de la irracionalidad puede ser muy costoso para los agentes y las decisiones que toman durante su vida. 
El Modelo de Amor Racional -MAR- se ha propuesto como una medida que prevé la necesidad de estructurar procesos de racionalización que guíen la generación y desarrollo del enamoramiento, evitando la presencia de factores irracionales que desencadenen diferencias entre los agentes al momento de tomar decisiones. Esto es lo que ha motivado el presente artículo y el estudio empírico en el que se ha basado, buscando validar la relevancia de las aportaciones que plantea el MAR.

Aunque esta investigación arroja nueva luz al estudio del amor como un proceso comportamental capaz de ser objeto del escrutinio de la razón, está consciente de los limitantes, sobre todo de carácter metodológico, a los que se enfrenta. El hecho de que lo que aquí se analiza tenga una relación natural con elementos subjetivos de los individuos, da la posibilidad de ampliar tanto la población muestra como el análisis cualitativo propuesto por el segundo instrumento, sin embargo, y por razones de tiempo y posibilidades, no se ha podido gestar un proceso con dichas características. Aun así, se espera que los resultados obtenidos marquen el camino y sean de interés para futuras investigaciones en torno a esta línea de trabajo.

En conclusión, el presente artículo pretende ser una plataforma para nuevos estudios sobre la racionalidad de las emociones, específicamente sobre la posibilidad de plantear procesos de racionalización de las mismas. La motivación primordial, era demostrar que modelos como el MAR pueden tener una clara aplicabilidad, siendo la base de estudios de carácter empírico que muestren su relevancia y valides. De esta forma, se plantea que la experiencia amorosa puede llegar a ser un proceso que goce de cierta certeza y objetividad conforme mayor sea la atención en la estructuración causal de la misma, consiguiendo así que se dé pauta a una nueva visión del amor, que se separe de la irracionalidad a la que hasta el momento se le ha relacionado.

\section{Bibliografía.}

CANTO-SPERBER, M., (2001). "Amor". En: Diccionario de ética y de filosofía moral. México: Fondo de Cultura Económica, pp. 50-63.

ELSTER, J., (2001). "Sobre las pasiones. Emoción, adicción y conducta humana". Barcelona: Paidós Iberoamerica.

ELSTER, J., (2002). "Alquimias de la mente: Racionalidad y emociones". Barcelona: Paidós.

ELSTER, J., (2007). "La explicación del comportamiento social: mas tuercas y tornillos para las ciencias sociales". Barcelona: Gedisa.

FREUD, S., (2001). "Más allá del principio del placer". Buenos Aires: Amorrortu.

GALIMBERTI, U., (2002). "Diccionario de psicología". Ciudad de México: Siglo XXI editores.

KAMBOUCHNER, D., (2007). "Cartesian Subjetivity and Love". En: The concept of love in 17th and 18th century philosophy. Leuven: Leuven University Press, pp. 23-42.

LEWIS, C., (2005)." Los cuatro amores". Alcalá: Ediciones Rialp.

MORALES, C., (2004). "Personalidad e inteligencia". Fundamentos de Humanidades, V(10), pp. 6986.

SINGER, I., (1999). "La naturaleza del amor: Cortesano y romántico". tercera ed. México: Siglo XXI editores. 
Invest. pens. crit. (ISSN 1812-3864)

Vol. 5, No. 2, mayo-agosto 2017

pp. 37-52

VÁZQUEZ, J. C., (2012)."Créelo, tú puedes tener pareja y encontrar el amor". 1 ed. México: Panorama.

VÁZQUEZ, J. C., (2016). "La racionalidad del amor. Un análisis desde la teoría elsteriana de la racionalidad de las emociones". Ciencia Ergo-Sum, noviembre, 23(3), pp. 198-204.

VÁZQUEZ, J. C., (2016b). "Una aproximación al Hiperracionalismo Elsteriano y su relación con los Factores de la Personalidad". Methaodos.revista de ciencias sociales, 4(2), pp. 213-224. 